\title{
Mathematical Laboratories: A New Power for the Physical and Social Sciences
}

\author{
Glen J. Culler
}

The concept of a mathematical laboratory has been developing throughout the lifetime of computers. The capabilities made available in systems supporting these laboratories range from symbolic integration, differentiation, polynomial and power series manipulation; through mathematical simulation; to direct control experimental systems. About 1961 two trends, one toward what has become known as on-line computation, the other toward time sharing gained enough recognition to develop national support and subsequently have come to represent what is now known as modern computation. An on-line system provides interactive facilities by which a user can exert deterministic influence over the computation sequence; a time-sharing system provides a means by which partial computations on several different problems may be interleaved in time and share facilities according to predetermined sharing algorithms. For reasons of economy it is hard to put a single user in direct personal control (on-line. that is) of a large scale computer. It is equally or more difficult to get adequate computation power for significant scientific applications out of any small scale economical computer. Consequently, on-line computing has come to depend upon time-sharing as its justifiable mode of implementation. On the other hand, valuable on-line applications have formed one of the major reasons for pushing forward the development of time-sharing systems. At present, both efforts have reached such a stage of fruition that one finds many systems incorporating selective aspects of the early experimental systems of both types.

In this chapter, we will bring out some of the key features of highly interactive direct control systems that have implications for continuing design efforts aimed at furthering the development of experimental mathematical laboratories. We then present a brief description of the foundations of an existing facility at the University of California at Santa Barbara and illustrate its use in a typical application. Finally, we discuss some extension of the system which will provide deeper power for future experimental applications.

Reprinted from Interactive Systems for Experimental Applied Mathematics, Proceedings of an ACM symposium. Edited by Melvin Klerer and Juris Reinfelds. (C) 1968, Association for Computing Machinery, Inc. Published by Academic Press, Inc.

\section{The Operation-Operand Orientation for Direct Control}

A primary aspect of modern computation in contrast to early computation is that of direct user control of computational processes. This is sometimes confused with very early "open shop" computing, in which the user was his own operator, but actually there is no similarity. The fact that a user in direct control commands his own console is only superficially analogous to his being his un uperatior: the impurtant distinction lies in the program power for direct control associated with his console. This is relatively new and has required some software (computer programming) sophistication over and beyond that of "assemblers" and "compilers". At present, the logical structure required for direct control is well understood and can be simply described. Consider, first some examples of direct control with which we are all familiar; for example, a musician playing an instrument. a person driving an automobile, an operator running a crane, and a child on his skateboard (or surfboard, for that matter). In each instance, there is a lively dynamic situation in which the person is actively involved as the guiding force. A certain amount of prior training is required for success and the variance in training and skill over all people is pronounced. Also, in each case, thoughtful designers have attempted to improve the performance capabilities through the nature of the controls provided. But no matter how well designed a piano may be, it is not possible for all people to play equally well. It may be the case that, the greater the control facilities, the larger the deviation in final performance. Thus, the essence of design quality should lie in the ultimate performance capabilities under talented control rather than in the elementary creature convenience for the initial learning period of the training. This is not to say that learning difficulties should be ignored, but that ultimate power should not be sacrificed for early convenience.

To design a direct control system, we first isolate the controlling elements (the operators) and their effects, (the transformation of the operands) and then provide a means for their interactive combination. For the design to be really successful, a deep understanding of the nature of the users and their performance desires is required. In the context of human involvement, the existence of Stradivarius violins and the yearly change of automotive design attest to the difficulty of the total direct control problem.

Abstracting from the examples above, we see that a logical structure for direct control consists of a set of operations together with a set of appropriate operands on which the user (a performer, driver, or athlete) can determine which operators 
are to be applied to which operands during dynamic, real-time activity. If there, are no operators that make it possible to concatenate sequences of other operators, the system is said to be manual; when there are concatenation operators, the system is said to be programmable and a program is precisely a concatenation of operators with specified operands. If the operands must be absolutely specified at the time the concatenation is formed (i.e.. when the program is constructed) then the system is absolutely programmable; if the operands may be specified by variables. then it is variably programmable; if the operators may be specified by variables. then it is substitutionally programmable.

As regards the selection of definitions of particular operators for a given application, very little can be generally said beyond the statement that the designer should try very hard to isolate the most maneuverable set of operators as primitives: this tends to shorten and simplify the prugrams ubuined through concatenation. But there are some observations we can make pertinent to the selection of operators that are independent of the applications area.

RESET

If there is a concatenation operator, then there should also be a reset operator which can override all other operators and force a stable halt, thus returning the system to manual control.

TEST

There should be some from of logical operation that permits a conditional selection of a successor (in computer terminology this is called a brance instruction).

ENTER

There should be some form of communication operator whereby the user can insert manual responses to program requests without REPEAT destroying the requesting program's control.

A fixed sequence of operations should be able to be recalled and performed any specified number of times.

Of course, we do not suggest that there is only one way of defining and utilizing the above facilities. Indeed, they can be very simple and minimal in capability or elaborate and extensive according to taste; but if the system does not have at least a minimal form of each of the above, then if suffers substantial restriction of its ultimate power.

Concerning concatenation operators, we have found it convenient to include several types within the same system. The reason for this is that with distinguished concatenations we are able to provide embeddings of program control that enrich the logical structure of the system. We'll return to illustrate this point later when we discuss console programming.

As a means to give specific explanation of how direct control systems can be used in mathematical laboratories, we now formulate an operational calculus in the area of discrete analysis and then detail its implementation in a direct control system. Following this, we will show its application in a laboratory system for the study of speech wave forms and finish the chapter with a brief description of the extension to more complicated data structures and associated operations.

\section{An Operational Calculus for Discrete Analysis}

The set of operations defined below have been chosen for their simplicity and ease of combination and have with-stood the test of experimental usage for several years and in several systems. The operands include single numbers, lists of numbers (one-dimensional arrays), double lists of number (two-dimensional arrays) and matrices; all of these are allowed to be either real or complex. Since the operands are of several categories, we must include a means for specifying the category. One way to do this is to set aside special variables for each category as illustrated by the expression:

$$
f(x)=\sum_{j=0}^{\infty}\left(\sum_{k=1}^{j} A_{j k} e^{-k x}\right) \frac{x^{j}}{j !}
$$

which shows the almost unwritten but broadly accepted rules of mathematical notation. THis may well be the best approach when the range of categories is properly restricted and when the conventions of a particular problem area are adhered to. But when many application areas utilize the same system each with their own notational habits, then this approach becomes unwieldy, though certainly not impossible. We select another approach which has been quite successful in practice. This consists of explicitly declaring the category; that is, of providing category operators:

REAL Operands shall be real.

CPLX Operands shall be complex.

I Operands denote single numbwers.

II Operands denote one-dimensional lists of numbers.

III Operands denote two-dimensional lists of numbers.

IV Operands denote matrices.

The operators are defined in relation to the operands as detailed below; they can be grouped as local and global by the nature of their definitions; local operators can be further grouped as arithmetic or as elementary functions. The main content of the definitions is best illustrated for II REAL; that is, for operators on one-dimensional real arrays. Thus in the definitions below, let each letter $A, \ldots Z$ denote a list of $n$ components

$$
A=\left(a_{1}, a_{2}, a_{3}, \ldots, a_{n}\right)
$$

such that each component [figure] $\mathrm{n}$ is substituted by a real number prior to any actual computation.

\section{A. Operator Definitions for II REAL}

1. Local operators are those which operate local to a coordinate index. This means increasing or decreasing the number of coordinates would not essentially change the consequence of these operations at earlier coordinate positions. 
a. The arithmetic operators consist of $\oplus, \odot, \odot$,

D, SQ, SQRT, NEG, INV, DIFF, SUM, MOD:

$$
\begin{aligned}
& X \nrightarrow Y=\left(x_{k}+y_{k} \mid k=1, \ldots, n\right) \\
& \mathrm{X} \fallingdotseq \mathrm{Y}=\left(\mathrm{x}_{\mathrm{k}}-\mathrm{y}_{\mathrm{k}} \mid \mathrm{k}=1, \ldots, \mathrm{n}\right) \\
& X \bigodot Y=\left(x_{k} \cdot y_{k} \mid k=1, \ldots, n\right) \\
& x \odot \mathrm{x}=\left(\mathrm{x}_{\mathrm{k}} \% \mathrm{y}_{\mathrm{k}}, \text { if } \mathrm{y}_{\mathrm{k}} \neq 0 ; 0 \text {, if } \mathrm{y}_{\mathrm{k}}=0 \mid \mathrm{k}=1, \ldots, \mathrm{n}\right) \\
& \text { se } y=\left(\mathrm{y}_{\mathrm{k}}^{2} \mid \mathrm{k}=1, \ldots, \mathrm{n}\right) \\
& \text { SQRT } Y=\left(\mathrm{y}_{\mathrm{k}}, \text { if } \mathrm{y}_{\mathrm{k}}>0 ; 0, \text { if } \mathrm{y}_{\mathrm{k}} \leq 0 \mid \mathrm{k}=1, \ldots, \mathrm{n}\right) \\
& \operatorname{NEG} Y=\left(-y_{k} \mid k=1, \ldots, n\right) \\
& \text { INV } Y=(1 \Leftarrow \mathcal{Y} Y) \\
& \text { DIFF } Y=\left(y_{k+1}-y_{k}\left|k=1, \ldots, n-1 ; 2 y_{n}-3 y_{n-1}+y_{n-2}\right| k=n\right. \\
& \operatorname{suM} Y=\left(\sum_{j=1}^{k} y_{j} \mid k=1, \ldots, n\right) \\
& \text { MOD } Y=\left(\left|y_{k}\right| \mid k=1, \ldots, n\right)
\end{aligned}
$$

b. The elementary function operators consist of SIN, $\cos$, LOG, EXP, ATAN, ARG, DEL.

$$
\begin{aligned}
& \operatorname{stn} Y=\left(\operatorname{sIN} y_{k} \mid k=1, \ldots, n\right) \\
& \cos Y=\left(\cos y_{k} \mid k=1, \ldots, n\right) \\
& \text { LOG } Y=\left(\text { In } y_{k} \text {, if } y_{k}>0 ; 0 \text {, if } y_{k} \leq 0 \mid k=1, \ldots, n\right) \\
& \operatorname{EXP} Y=\left(e^{y_{k}} \mid k=1, \ldots, n\right) \\
& \text { ATAN } Y=\left(\operatorname{TAN}^{-1} y_{k} \mid k=1, \ldots, n\right) \\
& \text { ARG } Y=\left(\pi \text {, if } y_{k}<0 ; 0 \text {, if } y_{k} \geq 0 \mid k=1, \ldots, n\right) \\
& \text { DEL } Y=\left(1 \text {, if } y_{k}=0 \text { or if } y_{k} y_{k+1}<0 \text { and }\left|y_{k}\right| \leq\left|y_{k+1}\right|\right. \\
& \text { or if } \mathrm{y}_{\mathrm{k}} \mathrm{y}_{\mathrm{k}-1}<0 \text { and }\left|\mathrm{y}_{\mathrm{k}}\right|<\left|\mathrm{y}_{\mathrm{k}-1}\right| \text {; } 0 \text {, if other- } \\
& \text { wise } \mid \mathrm{k}=1, \ldots, \mathrm{n} \text { ) }
\end{aligned}
$$


2. Global operators are those which do not operate local to coordinate indices and are those for which increasing or decreasing the number of coordinates would effectively alter the consequences of the operation at earlier coordinate positions. These are LS, RS, REFL, MAX, ID, EVAL;

$$
\begin{aligned}
& \text { LS } Y=\left(y_{2}, y_{3}, \ldots, y_{n}, y_{1}\right) \\
& \text { RS } Y=\left(y_{n}, y_{1}, y_{2}, y_{3}, \ldots, y_{n-1}\right) \\
& \text { REFL } Y=\left(y_{n}, y_{n-1}, \ldots, y_{2}, y_{1}\right) \\
& \text { MAX } Y=\left(\operatorname{MAX}_{j=1} y_{j} \mid K=1, \ldots, n\right) \\
& \text { ID } Y=\left(\frac{2 k-n}{n-1}-1 \mid x=1,2, \ldots, n\right) \\
& (X, Y) \text { EVAL } U=(U, Y(U)) \text { to first order. }
\end{aligned}
$$

This later operation requires further specification. First extend $\mathrm{x}$ b) augmenting with $\mathrm{x} \cdot=-\infty$ an $1 \mathrm{i}_{n+i}=+\infty$

$$
\begin{aligned}
& -\infty \leq t \leq M_{k=1, \ldots . n} X(t)=Y\left(x_{\text {min }}\right) \\
& \operatorname{MAX}_{k=1, \ldots, n} t \leq \infty ; Y(t)=Y\left(x_{\max }\right)
\end{aligned}
$$

Finally, for each value of $U$, (i.e., $u_{j}, j=1, \ldots, m ; m$ not necessarily equal to $n$ ) determine $x_{\mu}$ and $x_{\nu}$ such that

$$
\begin{aligned}
& x_{\mu}=\left(\begin{array}{l}
\operatorname{MAX}_{k=0, \ldots, n+1} \mid x_{k} \leq u_{j}
\end{array}\right) \\
& x_{\nu}=\left(\begin{array}{l}
M \mathbb{N} \\
k=0, \ldots, n+1
\end{array} \mid x_{k}>u_{j}\right)
\end{aligned}
$$

and choose

$$
y_{j}=y_{\mu}+\frac{u_{j}-x_{\mu}}{x_{\nu}-x_{\mu}}\left(y_{\nu}-y_{\mu}\right) \ldots
$$

In the exceptional case when some of the $u_{j}$ 's fall outside the range of $X$, the extension of $Y$ given above gives $Y$ one constant value to the left of $x_{\min }$ and another constant value to the right of $x_{\max }$. Also, notice that no ordering of $x$-values or the 8-values has been assumed; this is important for the required generality of the EVAL operator. As defined, it provides a basis for graphical composition of functions and makes it possible to change discrete variables in the middle or a calculation. When some of the. functions occurring in a computation are only known discretely and not at the same intervals used in the calculation, then some form of this EVAL operator is mandatory.

\section{B. Extension of II CPLX}

The extension of II REAL operators defined above to the II CPLX operators is accomplished by first introducing complex lists:

$$
z=X+1 Y ; \quad g_{k}=x_{k}+1 y_{k}, k=1, \ldots, n
$$

and then defining the arithmetic operators using complex arithmetic on corresponding coordinates. All this is immediate up to the selection of branch cuts as required in the definition of LOG and SQRT. The best way we have found for maintaining compositional compatibility is to define ARG with the facility of branch specification and define all other functions requiring branch definitions in terms of ARG, If we think of a one-dimensional complex array as an ordered set of vertices of a polygonal arc in the complex plane, then the change in argument at adjacent vertices is not more than $2 \pi$, consequently, we can computer this change using either the positive or negative principal branch of the argument function. In the event that one of our vertices $\left(\Phi_{k}\right)$ actually coincides with the origin, we augment that array and replace that vertex with two new vertices $\left(\Phi_{\mathbf{k}}, \Phi_{\mathbf{k}}\right)$ ) chosen on the adjacent polygonal segments.

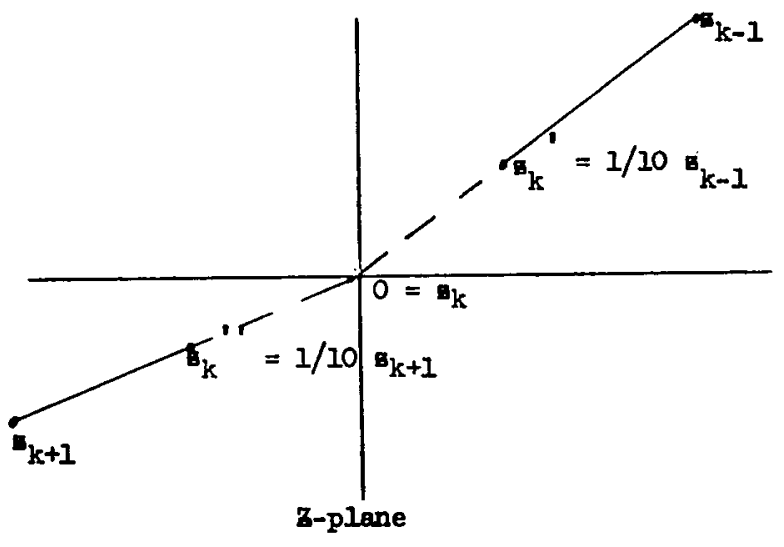

The final argument function, $\mathrm{ARG}_{+}$or ARG. is just the II REAL SUM of the argument difference. Of course, both ARG and MOD of II CPLX are real valued functions, thus part of their calculation consists of making their imaginary parts equal to zero. Also, because conjugation plays such an important role in complex structures, we choose to drop the NEG operator and replace it with *, complex conjugation. Since the real operators when composed turn out to be transformations on Y-coordinates, both NEG and * have the same result: the change in sign of the $Y$-coordinates.

The global operators also extend easily; LS and RS are direct, REFL brings in a new facility

Let

$$
\begin{aligned}
& \mathrm{Z}=\mathrm{X}+\mathrm{iY} \mathrm{X}, \mathrm{Y}, \mathrm{II} \text { REAL } \\
& \text { REFL } \mathrm{Z}=\operatorname{REFL}(\mathrm{X}+\mathrm{iY})=\mathrm{Y}+\mathrm{iX},
\end{aligned}
$$

thus exchanging real and imaginary parts. Coupled with REAL REFL we can still quickly reverse the orderig of a complex array

The extension of MAX results in:

$$
\operatorname{MAX}(X+i Y)=\operatorname{MAX} X+i \text { MAX Y X,Y II REAL }
$$


The extension of EVAL to II CPLX is not well defined; for it to be so requires strenuous conditions on the arc at which the initial pair is being evaluated. and the operation does not appear to be computationally valuable. The situation is much improved. however, for EVAL III CPLX, which we will discuss later. The operator ID is a fundamental array generator; for II REAL. it produces an interval from -1 to +1 with $n$ equally spaced values; for II CPLX it produces selectively a discrete representation of the unit circle (i.e., the nth roots of unity) or a square about the origin joining $(-1-i, 1-i, 1+i,-1+i)$ with equally spaced points.

\section{Contraction to I REAL and CPLX}

Even in problems that are primarily array oriented, we frequently need to use and produce scalars, both real and complex. Consequently, part of our operational calculus consists of operators of category [. The definitions of these operators are obtained by setting $n=1$ for category II operators and discarding those that lose their meaning; as for example. SUM, DIFF, MAX. LS, RS, REFL, EVAL, etc.

Starting from this bare frame of operators. we must now show how to accomplish significant control of a computer system and obtain the facilities of a mathematical laboratory.

\section{Construction of a Mathematical Laboratory}

To build a system that under direct control performs the operations just defined we first introduce a keyboard oriented especially to those operations. Each time one of the keys is depressed a number uniquely corresponding to that key is transmitted to our computer and stored in its memory. The program within the computer analyses this number and determines the type of key just depressed, then responds in accordance with conditions already set up by the antecedents of this key. We make this explicit for our operational calculus. First. ubserve the key buard in Figure 1 . It is divided into an upper-half and a lower-half; we designate the upper-half as operators and the lower-haif as operands. The number transmitted by depressing a key has two parts, the first is the name of the keyboard and the second is the number of the operator key or operand key. These are, of course, binary numbers and are chosen to agree with the American Standard Code for Information Interchange. The format of the number stored in the computer is:

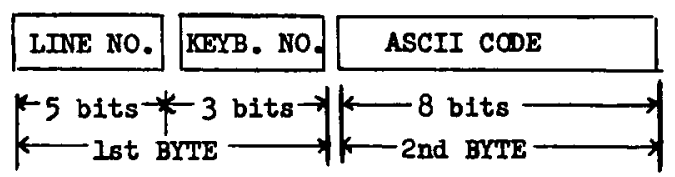

where the line number refers to the current incoming telephone connection is inserted by the equipment servicing the telephone lines, the keyboard number and the ASCII code for the depressed key actually come in on the telephone line. If no telephone lines are used, that is if the keyboard is physically near the computer, then the line number is taken to be zero.

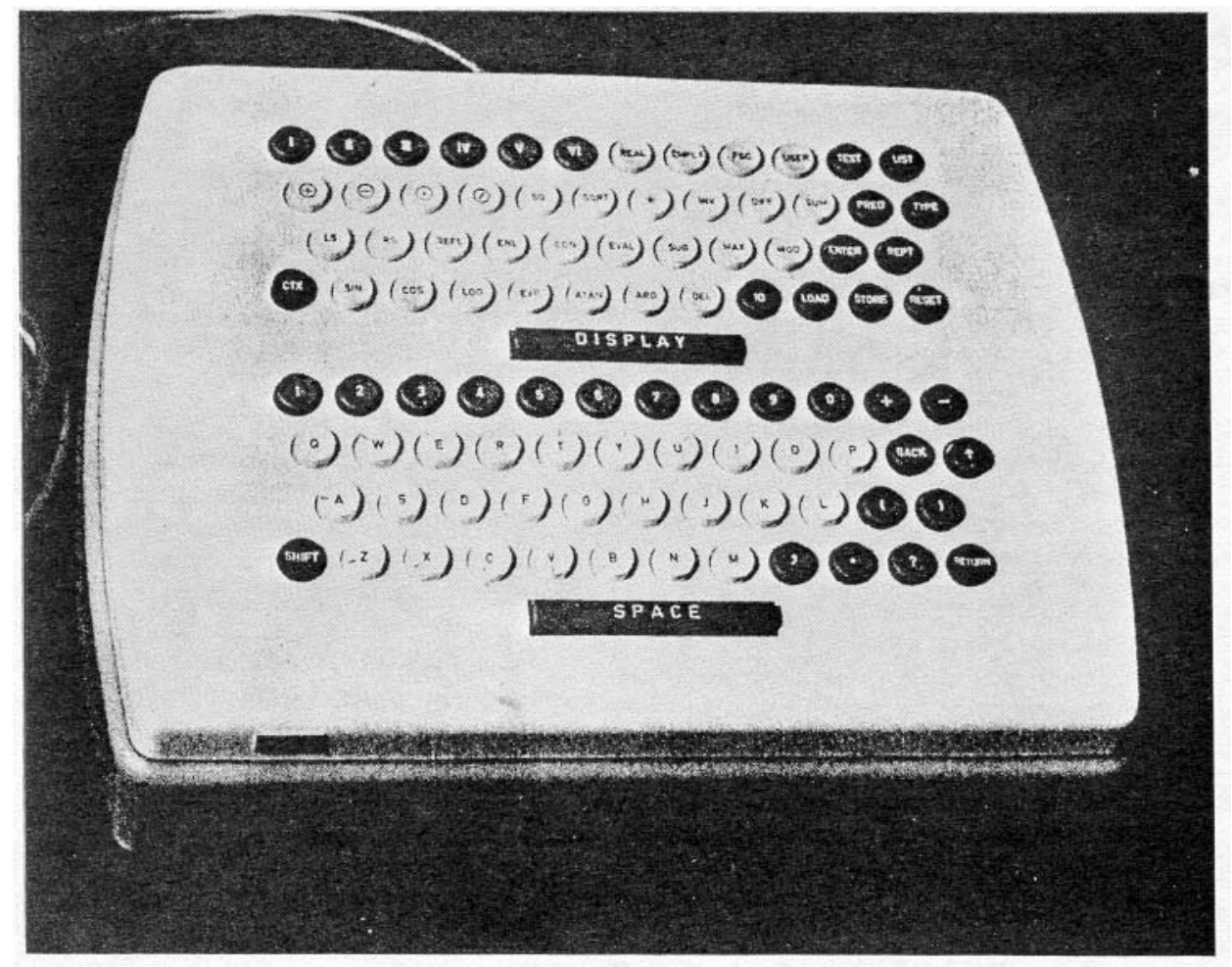

Figure 1. An operational calculus keyboard for discrete anaiysis. 
The operators are grouped according to their system properties and typical use:

A. Operator Category Keys - Blue I, II, III, IV, V, VI.

B. System Change Keys - Yellow REAL, CPLX, SYST, USER.

C. Program Control Keys - Green, Black, Red LIST, TEST, PRED, TYPE, ENTER, REPEAT, DISPLAY, ERASE, LOAD, STORE, RESET.

D. Application Operator Keys - White + * / SQ SQRT *NEG INV DIFF SUM LS RS REFL ID EVAL MAX MOD SIN COS LOG EXP ATAN ARG DEL.

The operand keys are also grouped according to their usage:
A. Numerical Keys - Blue
B. Alphabetic Keys - White
C. Punctuation Keys - Green
D. Typing Control Keys - Black

Using such a keyboard we can direct the activities of our computer by the sequence of keys depressed. But to do this well, we need to have an accurate mental image of what the computer does in response to each sequence. From the point of view of communication. this is analogous to reading or doing mental arithmetic or in feeling which note should be played next on a mosical insirument. The issues involved in bringing this about are threefold:

1. Understanding when an operator-operand sequence is informationally complete,

2. Knowing the family of operand specifications for each operator used.

3. Keeping track mentally of the consequences of each transformation performed.

It is clear that not everybody will do this equally well, but those who use direct control systems with power soon develop these capabilities. A different approach, which is analogous to pushing a bicycle instead of riding it, is to use interactive cues from the computer which force control to go along certain preprogrammed sequences. This is especially useful during the initial training period, but should be curtailed when the user's facility has developed. Systems with too much cueing soon become loggish and stultifying to the able user.

Our method for making the required mental images easy, is to associate a so-called working register with each operator category I, II, III, IV, etc. and picture the consequence of each informationally complete operation as being placed in this working register. As one changes operator categories, the contents of the associated working registers are not changed and hence upon returning to a given category, its contents remain as previously constructed. It is not necessary to prefix each operator with its category designation, that is, the operator category is maintained until a new category is specified. To be more specific about this, we discuss some of the operators in relation to their implied transformations of the working registers. Let the working register be denoted by [figure] and its two associated sub-registers by $\alpha$ and $\beta$. To distinguish register categories we use a category subscript:

$$
\begin{gathered}
\zeta_{\mathrm{I}}=\left(\alpha_{\mathrm{I}}, \beta_{\mathrm{I}}\right) \\
\zeta_{\mathrm{II}}=\left(\alpha_{\mathrm{II}}, \beta_{\mathrm{II}}\right), \text { etc. }
\end{gathered}
$$

Now on II REAL, a composition of functions like...

$$
f(x)=\log \sin \left(3 x^{2}+1\right)
$$

represented correctly by ...

$$
\left(X, \log \sin \left(3 x^{2}+1\right)\right)
$$

where $X$ is the independent variable running over its prescribed domain. To make such a set of ordered pairs, we construct the discrete domain for $\mathrm{X}$

$$
X=\left(x_{1}, x_{2}, \ldots, x_{n}\right)
$$

and put in $\alpha_{\mathrm{II}}$, then compose the three transformations and put the result in $\beta_{I I}$. We must be able to transfer data lists to and from both associated registers $\alpha$ and $\beta$ of all categories; this is one of the uses of the LOAD and STORE operators.

LOAD:

Move the data selected by the successor into the working register.

\section{STORE:}

Move the data from the working register into the location defined by the successor.

A typical manner of operation is to load some initial data into $B$, then to transform it by a sequence of transformations and finally to save it away by storing it as some dependent variable. The point of having LOAD and STORE directly available is to provide specific control over the contents of the working register. On the other hand, there are many instances where the transfers to and from the working registers can be performed implicitly and at a saving of manual labor. Thus, we make implicit transfers also possible where convenient. For II REAL, the operations defined in Section 2 all leave the consequences in $\beta_{I I}$, which satisfies the compositional conditions noted above. Each operation occurs in a number of situations according to is predecessors and successors, but within the operator sub-groups defined in Section 2A, above, there is sufficient similarity that is adequate for us to illustrate a typical member of each sub-group.

Computations are carried out in complete compositional sequences, that statement of completion being any category key, DISPLAY, STORE, or RETURN. After a computation is performed, the results are left in $\beta$, whether or not they have been saved by the calculation. A new compositional sequence can be initiated by an operator or operand; if initiated by a variable operand which is not immediately followed by $=$, then an implicit LOAD is effected; if the variable operand is immediately followed by $=$, then the composition sequence will end by effecting an implicit STORE associated with that variable.

To illustrate the above relations of compositional sequences, we analyses equivalent sequences of computing $F=\log \sin$ $\left(3 x^{2}+1\right)$. Assume that we are using II REAL and that $X$ has previously been constructed.
A. LOAD X SQ * $3+1$ SIN LOG STORE F
B. $3 \mathrm{X}^{2}+1$ SIN LOG $=\mathrm{F}$
C. $\operatorname{LOG} \operatorname{SIN}\left(3 X^{2}+1\right)=F$
D. $F=\operatorname{LOG} \operatorname{SIN}\left(3 X^{2}+1\right)$ 
These all make the results of computing:

$$
F_{k}=\operatorname{LOG} \operatorname{SIN}\left(3 X^{2}+1\right)
$$

available as the discrete data for the variable $F$, but they show different aspects of the direct control system. In a, the composition sequence is constructively ordered but greatly different in appearance from the original expression. After each step of $A$ the contents of $\beta_{I I}$ are explicitly known to the user:

$$
\begin{aligned}
& \text { LOAD } x \quad\left(x_{1}, \ldots, x_{n}\right) \in \beta_{I I} \\
& \text { se } \quad\left(x_{1}{ }^{2}, \ldots, x_{n}{ }^{2}\right) \in \beta_{\text {II }} \\
& \text { Q } 3 \quad\left(3 x_{1}{ }^{2}, \ldots, 3 x_{n}{ }^{2}\right) \in \beta_{I I} \\
& \text { (I) } 1 \quad\left(3 x_{1}{ }^{2}+1, \ldots, 3 x_{n}{ }^{2}+1\right) \in B_{\text {II }} \\
& \sin \quad\left(\sin \left(3 x_{1}{ }^{2}+1\right), \ldots, \sin \left(3 x_{n}{ }^{2}+1\right)\right) \varepsilon \beta_{I I} \\
& \text { LOG } \quad\left(\log \sin \left(3 x_{1}^{2}+1\right), \ldots, \log \sin \left(3 x_{n}^{2}+1\right)\right) \in \beta_{I I} \\
& \text { STORE F } \quad\left(\log \sin \left(3 x_{1}^{2}+1\right), \ldots, \log \sin \left(3 x_{n}^{2}+1\right)\right) \in B_{I I}
\end{aligned}
$$

In $\mathrm{B}$, we begin with an implicit LOAD, use the power operator $\uparrow$ which does not appear except in forming the superscript. and replace STORE with which is equivalent in this context. In $\mathrm{C}$, we use an interpretive ordering of the operators and obtain a familiar appearance as a formula but lose any apparent correspondence to the transformations performed. In $\mathrm{D}$, we precisely have mathematical notation and are satisfied to be sure that $F$ will end up with the right answers. In such simple circumstances it, of course, makes no real difference which approach is used. In practice, it depends upon the experimental requirements of the situation; that is, if there are no possible computational difficulties and all the compositional sub-sequences are unimportant to the user, then the automatic formula approach of D is most satisfying; but if something has gone sour, (say through singularities, bad distribution of data, or loss of accuracy through numerical pathologies) then it is important to be able to construct the results through step by step transformations of $\beta_{I I}$ and $A$ is the most satisfying. Since both circumstances occur frequently in using a mathematical laboratory, we must provide both facilities (B and $\mathrm{C}$ are irrelevant, they are simple variations of $\mathrm{A}$ and $\mathrm{D}$ ).

The final ingredient of the laboratory, is a display tube which permits visual understanding of the operations carried out by use of the DISPLAY operator and will be illustrated in the next section.

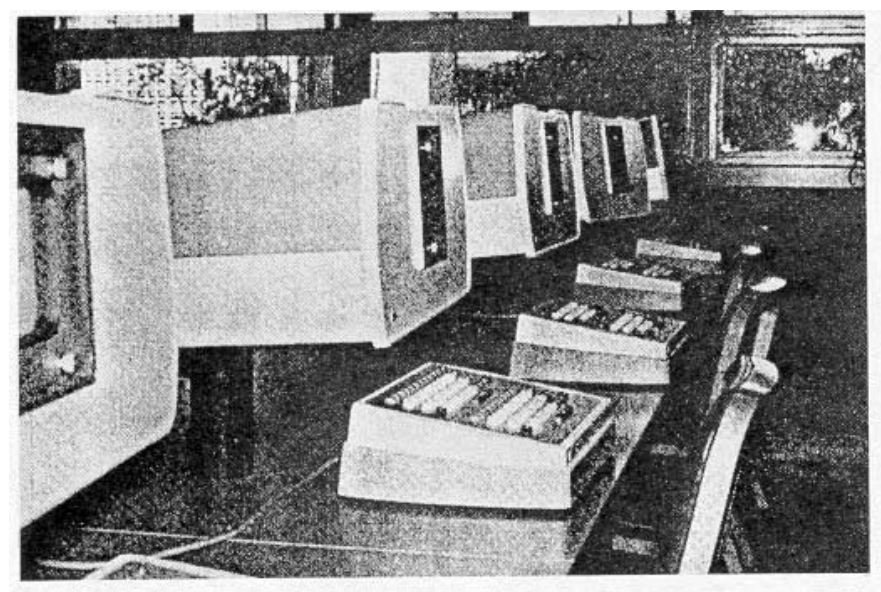

Figure 2. User consoles in the University of California, Santa Barbara mathematical laboratory.

\section{Experimental Analysis of Speech Wave Forms}

In a total system like that outlined above, there are so many operations each with several instances and special uses that we cannot present all contingencies and facilities short of producing a fairly large user's manual. But we can convey some sense of a user's experience to the reader by tracing through and explaining a brief but fairly typical actual sequence of operations as they occurred when we were studying how to analyze wave forms arising in natural speech. The equipment used consisted of an early mathematical laboratory built around the $\mathrm{RW}-400$ polymorphic computer with the addition of an IBM 1800 process control computer. To the latter computer we attached a microphone input and an audio speaker output as well as a two-way data transfer connection to the first computer. Following the operator-operand philosophy outlined in Section 2, we selected a set of primitives that gave us control of the microphone input and the speaker output and programmed them for the IBM 1800 . The control of these operations was implemented as a special category, VII, and added to the former mathematical system. The operations consist of controls for recording levels, for selecting which sub-lists of data to listen to, for selecting and transferring data to and from a back-up disk. Thus, we can digitally record live speech, bring it into the mathematically orienced system and decompose it by whatever means occur to us. In the other direction, we can manufacture mathematical functions and use them to build data lists in the IBM 1800; then make the 1800 output this list through an audio speaker for us to hear.

The data buffer, $\alpha$ VII, consists of 7440 memory locations subdivided into 60 blocks of 124 numbers each. We chose to use some letters on the alpha-numeric keyboard followed by specification of blocks and position within the blocks as non-numerical operations.

\section{A. Category VII Operators}

\section{RETURN:}

Initiate operation just specified.

R k:

Begin recording input data when the signal exceeds the threshold $k$ ( 30 threshold levels are available).

Q:

Quiet, cease output to the speaker.

L a, b, c:

Listen; initiate output to the speaker starting with block $a$, position $c$, and output $b$ consecutive blocks before delay.

D a:

After each speaker output delay a times a unit interval before repeating the output.

I a,p:

Input 1 block starting at block a, position $c$ and place the data in $\beta_{\text {II }}$ of the RW -400 system. 
O a,c:

Output 1 block from $\beta_{\text {II }}$ of the RW-400 system and put it in block a starting at location $\mathrm{c}$.

S k:

Apply a k-point smoothing formula to that part of the data list in $\alpha$ VII addressed by the last $L$ a,b,c operator.

LOAD k:

Transfer the data on disk cylinder $\mathrm{K}$ into $\alpha_{\mathrm{VII}}$ of the 1800 .

STORE $\mathrm{k}$ :

Transfer the data list in $\alpha_{\text {VII }}$ of the 1800 .

LS:

Specify a variable to be used as a vector of scales for the blocks of $\alpha$ VII.

RS:

Rescale all blocks to a common scale.

\section{B. Experimental Analysis of $S A T$}

The pictures below showing a grid were taken with a Polaroid camera attached to a Tektronix storage oscilloscope that records the analog voltage signal output from the IBM 1800 . The others not showing a grid were taken with the same camera attached to a second such oscilloscope used as a display device for alpha-numeric and graphical output from the RW-400.

To initiate a run, the user presses

\section{RESET LOAD USER}

and receives the computer's response requesting his user number. After the number is provided, the computer retrieves his complex of user programs and accumulated data and types out the user's own system message.

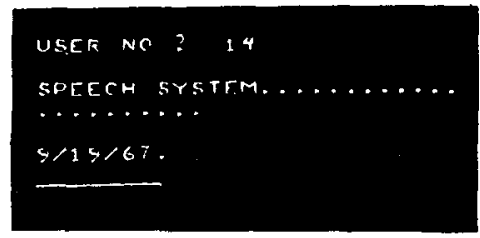

Figure 3. Start of a run

To record a word for study, we pressed

\section{R 7 RETURN}

and pronounced "sat" into the microphone. After adjusting the oscilloscope scales as shown below the graph, we see the complete wave form of the recorded word.

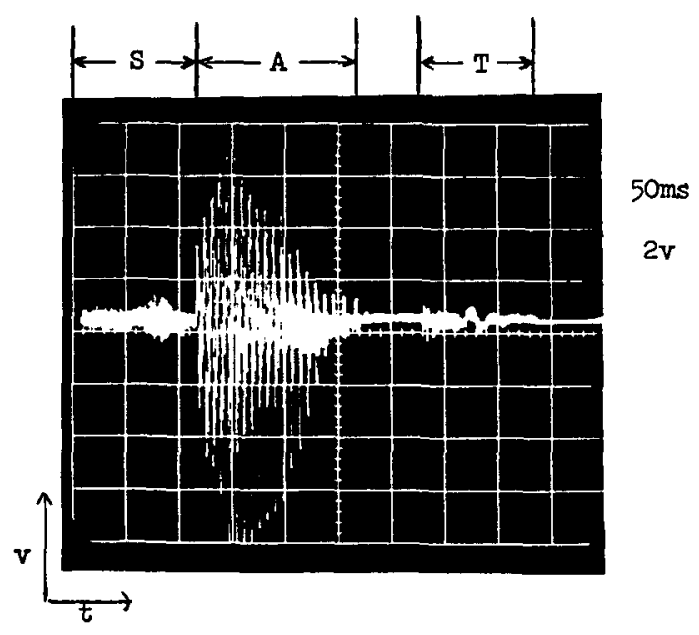

Figure 4. Wave form of SAT

In order to view the character of the wave form producing $S$ we pressed

\section{1. 17 RETURN}

and adjusted as is shown. Since the oscillations were too rapid to see we then pressed

\section{9, I RETURN}

which allowed us to see block 9 alone. where the large amplitude of S occurred. Compressing the time scale resulted, finally, in a good picture of the mixture of wave functions in S.

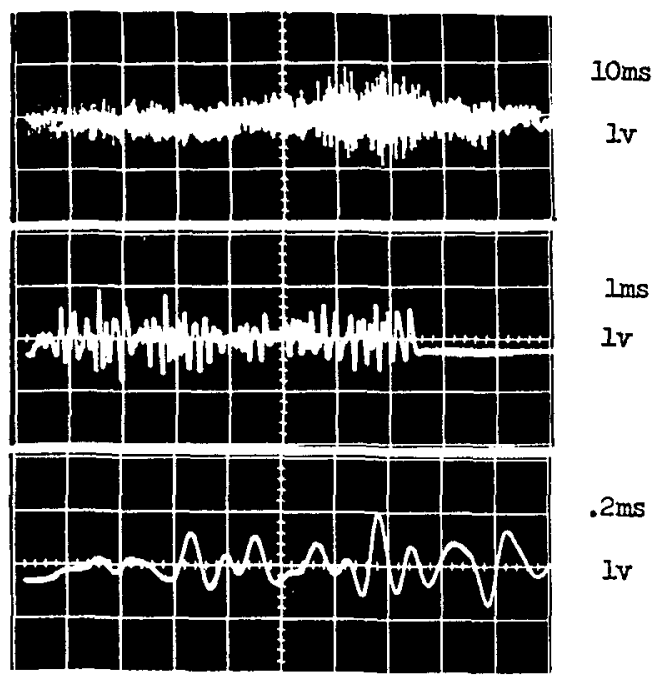

Figure 5. Details of the $S$ wave form

Carefully observing the form of the last graph presented above allows one to identify the actual ingredients of $\mathrm{S}$ as a sequence of Gaussian wave rumiluns. i.e. nommalized deritatives of

$$
\operatorname{EXP}\left(\left(-t^{2} / 2 \sigma^{2}\right)\right.
$$

having an assortment of $k$-values (the order of the derivatives) and occurring haphazardly in time. This decomposition of speech wave forms into Gaussian wave function components is a new approach to the classification, analysis and synthesis 
of speech elements and, of course, cannot be properly treated here. But we can illustrate a simple instance of it. For this purpose, we will study the data producing the A in SAT.

The three pictures of Figure 6 were obtained respectively by
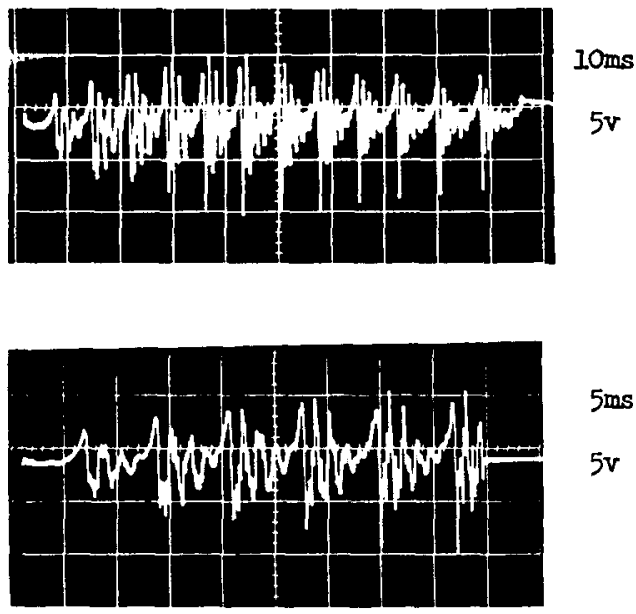

$5 v$

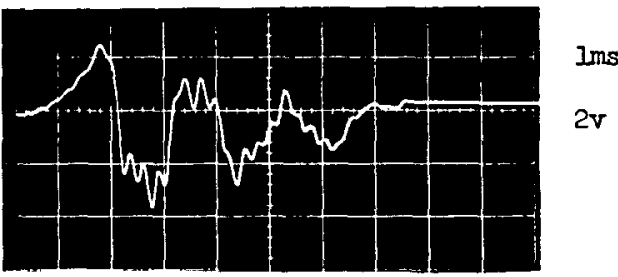

Figure 6. Pitch periods of the $A$ in SAT

\section{14, 14 RETURN}

L 14, 6 RETURN

\section{L14, 1, 70 RETURN}

and show a successive blow-up of the initial pitch period of the A (the wave form lying between the larger extremes in a nearly recurrent manner is called a "pitch period"). From the first of these graphs, we can observe a relative rise in the second and third positive extremes. The fourth pitch period seems to be fairly representative of this family, so we all it into the mathematical system by pressing

$$
\text { I } 18,50
$$

still of category VII, then enter category II REAL for the ensuing analysis, as shown in Figure 7 , we then put the curve on maximum scale by adding zero,

$$
+0
$$

and then save it as the variable operand $Q$ and finally display the curve. Each such pitch period is made up of essentially three signals distinguished by their oscillations frequencies. There are many ways to attempt to separate out these sub-signals: we shall use the DIFF operator in combination with an integral mean smoothing operator as in VII S.K above, or a five point smoothing operator as shown in Figure 8. The graph in Figure 8 is the result of applying two successive smoothings. Subtracting this smooth function gives a first approximation to the high format.
Due to inherent properties of smoothing operators, we cannot be sure that this approximate formant is pure; that is, it may still contain some components of the two low formants. Running it through the same smoothing process results in a function of small amplitude.

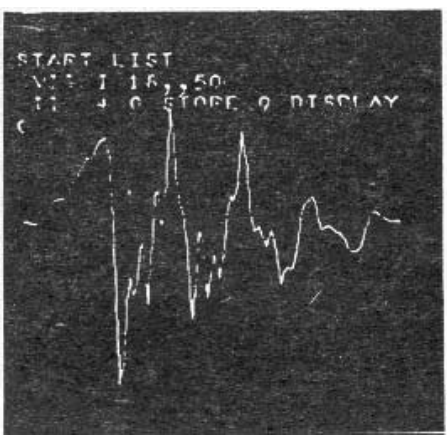

Figure 7. The fourth pitch period of A in SAT

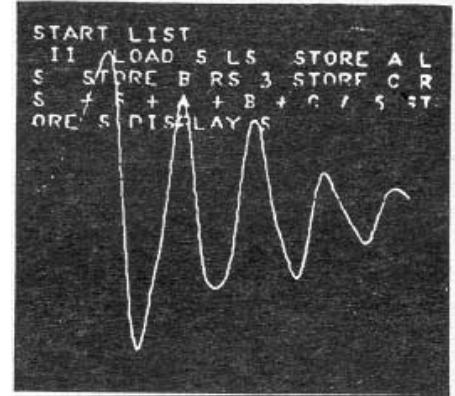

Figure 8. Combination of Low Formants

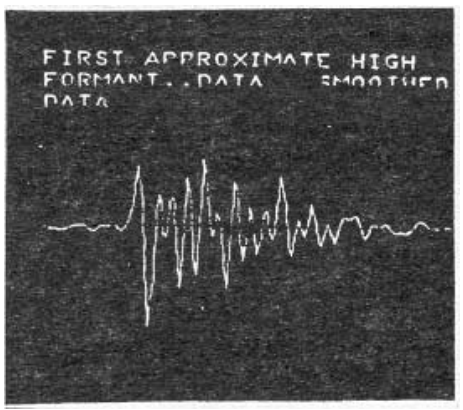

Figure 9. Approximate High Formant

When this function is subtracted from that shown in Figure 9. we obtain the extracted high formant as shown in Figure 10.

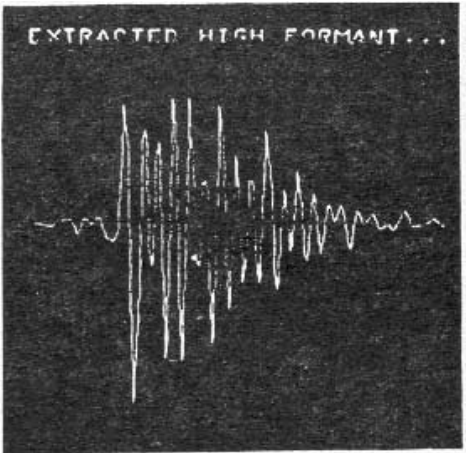

Figure 10. Second approximation to the high formant 
Another method based on the DIFF operator uses the fact that for well separated frequencies, and fairly close data points, the difference operator separates the relative amplitudes of the formant. Applying DIFF DIFF NEG to the original data curve of Figure 7, produces the graph shown in Figure 11. The normalization of this function is lost in the DIFF process, so only shapes should be compared.

Returning to the consideration of the low formants, we can use DIFF again to try to split apart this pair. The dotted graph in FIgure 12 is the smoothed graph above, and the solid graph is its DIFF. Notice particularly the disfiguration of the solid graph near the large positive maximum, this shows where two wave functions are overlapping.

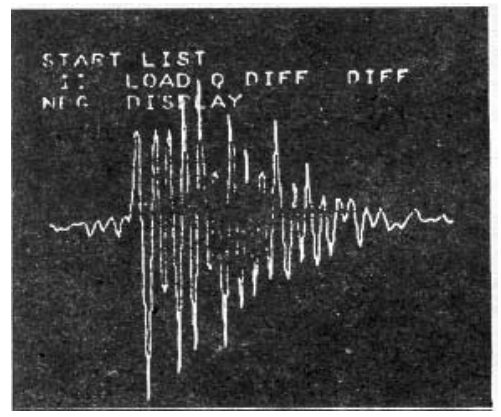

Figure 11. Unnormalized approximate high formant using the DIFF DIFF operator

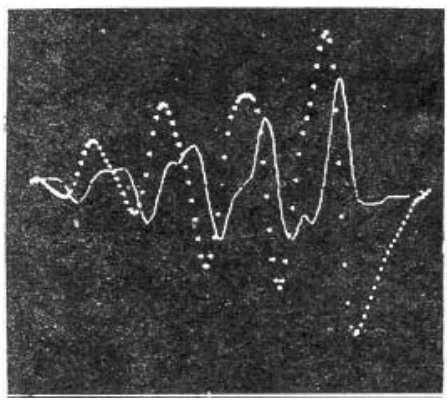

Figure 12. DIFF applied to the combined low formants

Our approach now is to identify the wave functions in this difference graph and compose them to approximate it, then to use the SUM operator to obtain an approximation of the dotted graph of Figure 12. Experimenting with the Gaussians width results in the choice of

$$
1 / 2 \sigma^{2}=81
$$

as one which looks hopeful. To see this compare figure 13 with the shapes of the extremes in Figure 12. Our conjecture is that for this A sound, the deep first minimum results from the second derivative of the Gaussian and the second wave function occurring later is just its normalized derivative. Using the LS and RS operators we position the minimum and with NEG, MAX. INV we choose amplitudes and thus construct the wave functions shown in dotted curves of the first two graphs of Figure 14. Adding the resulting wave functions together, we next apply DIFF and then reposition and renormalize the result to fit the second pair of extremes as shown in the dotted graph of the third picture. Continuing this process once more then completes our approximation and using SUM results in the dotted graph of Figure 15, which is compared to the solid curve resulting from the smoothing operator. Now our approximation has no components of the lowest formant in it which accounts for the changes in elevation on the right hand side of Figure 15. Finally, adding in the high formants allows comparison of the original data curve with our fit.

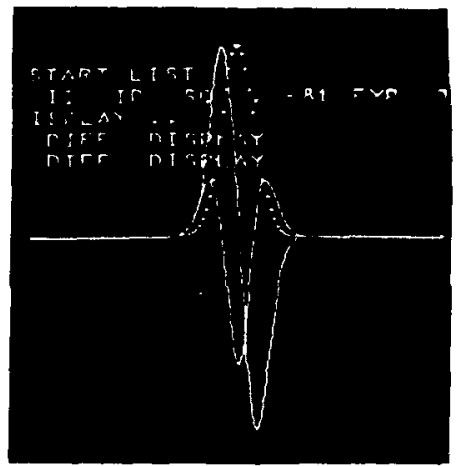

Figure 13. The first three Gaussian wave functions
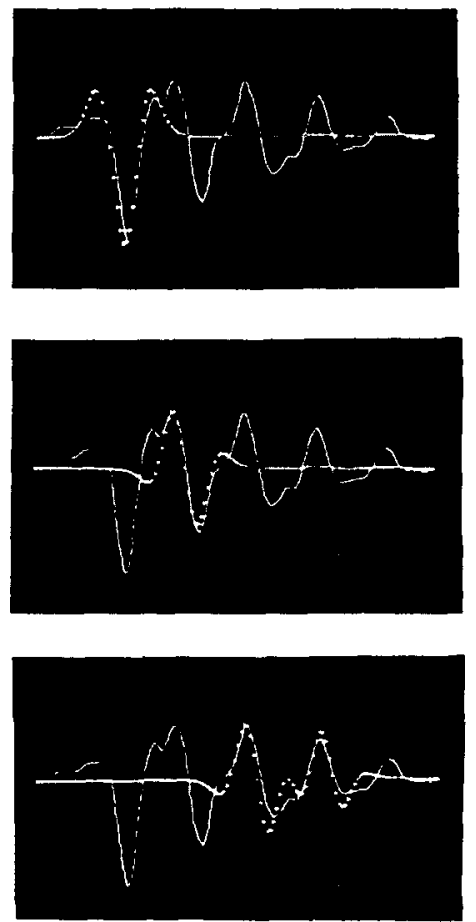

Figure 14. Fitting the difference curve by wave functions

If one looks carefully back at the extracted high formant, he will see one of its constituent wave functions. The procedure just outlined will provide a representation in the same manner and result in a total wave function representation of the original data curve.

Going back to the speech computer, we use

\section{L t0. 19 RETL'RN}

and get a good view of the wave making the $T$ sound. Since we are always able to see and hear the data simultaneously, the identification of the linguistically effective parts of the wave forms is easily made. The data for $\mathrm{T}$ is similar to that 
for S except for the single large slow wave function clearly evident in the $T$.

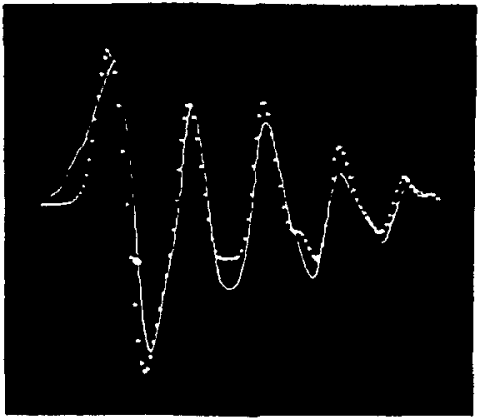

Figure 15. Comparison of smoothed data and wave function fit

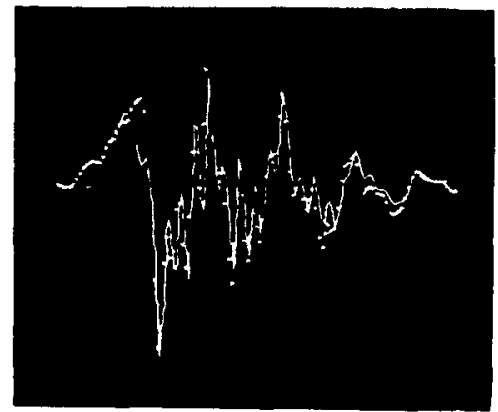

Figure 16. Comparison of original data and wave function fit

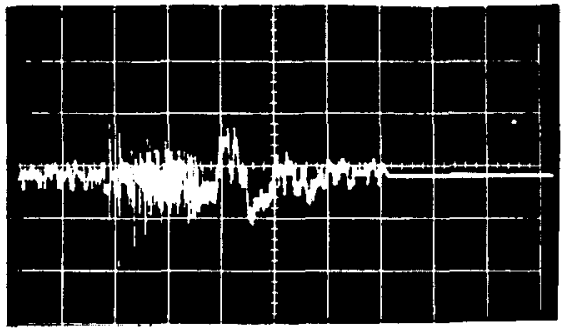

Figure 17. The wave function for the $T$ in SAT

\section{Extension or Higher Category Operations}

The requirements of a structure supporting operations of categories II and IV both REAL and CPLX can be made clear by formulating several problems that point the way towards generalized data objects and their associated transformations.

\section{A. Problems for Category III REAL}

1. Integral equation

Let $s, t$ be independent variables running over an interval $[a, b]$ and $F(s, t, u)$ a function of three variables such that for each point $(s, t) \varepsilon[a, b]^{2}$. $F$ is a rational function of $\mathrm{U}$. For some class of non-zero driving functions $g(t)$ we wish to determine $U(t)$ such that

$$
U(t)=g(t)+\int_{a}^{b} F(s, t, U(s)) d s .
$$

To check whether a given function $U(t)$ satisfies this equation the coefficient functions $A_{j}(s, t), B_{j}(s, t)$ must be computed and the rational function of order $\mathrm{J}$ constructed.

$$
F(s, t, U(s))=\frac{\sum_{j=0}^{J} A_{j}(s, t) U^{j}(s)}{\sum_{j=0}^{J} B_{j}(s, t) U^{j}(s)}
$$

If we choose III REAL precisely as a two-dimensional extension of II REAL, and use discrete variables

$$
\begin{aligned}
& S=\left(s_{1}, s_{2}, \ldots s_{n}\right) \\
& T=\left(t_{1}, t_{2}, \ldots t_{n}\right)
\end{aligned}
$$

then the data structure for the coefficient functions

$$
A_{j}(s, t)=\left(A_{j}\left(S_{k}, t_{i}\right) \mid k=1, \ldots, n: l=1, \ldots, n\right)
$$

are the directly addressable operands for III REAL. The extension of the II REAL operators is achieved by considering the columns of III REAL operands (or rows) to be of the same class (but differently labelled) as II REAL operands. Then II REAL may be used sequentially to build III REAL operands.

2. Properties of surfaces over plane domain

Let $H(x, y)$ be defined for points $(x, y)$ on the closure of a domain $\mathrm{D}$ which consists of the interior of a simple closed curve $\Gamma$

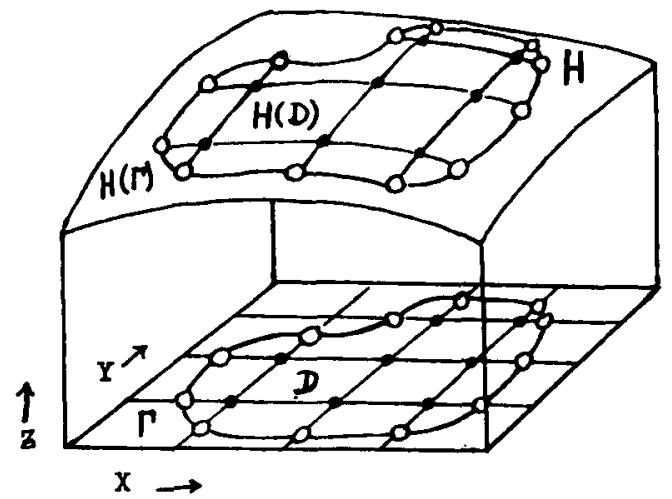

Figure 18. Surface representation of III REAL operands

Since D is not necessarily rectangular we must enrich our data representation by some form of logical selection which permits partial columns and partial tows to be easily used. Now let

$$
\begin{aligned}
& X=\left(x_{1}, \ldots, x_{n}\right) \\
& Y=\left(y_{1}, \ldots, y_{n}\right)
\end{aligned}
$$

be two REAL operands. An operator $W$ which forms the product set $X \times Y$ builds a discrete rectangular domain which is a natural operand for III CPLX, since corresponding to each index pair $(\mathrm{j}, \mathrm{k})$ there is a coordinate pair $\left(\mathrm{x}_{\mathrm{j}}, \mathrm{y}_{\mathrm{k}}\right)$ which can be thought of as a 
point on a plane grid. Observing that $\Gamma$ can be represented as a II CPLX operand, we let

$$
X \times Y=W \text {. III CMPLX }
$$

and

$$
\Gamma=\mathrm{G}, \quad \text { II CMPLX }
$$

then on III REAL we generate a real array of l's and $O$ 's which is the characteristic function of the interior of $G$ with respect to W:

\section{LOAD III CPLX W DEL II CPLX G}

where $G$ is insured to be closed by considering the first and last points to be adjacent vertices in a closed polygon. This characteristic function can be used as a logical mask in calculations involving $\mathrm{D}$; also, it is binary by nature, so it can be compressed to bit by bit representation and carried along with $W$ above as the representation of D. As shown in Figure 18 above, it is also convenient to interpolate $\Gamma$ at the grid line crossings of the $\mathrm{W}$ array. This is accomplished on II CPLX by:

\section{LOAD G EVAL III CPLX W}

and results in a new representation of $\Gamma$ consisting precisely of the interpolated intersections of the grid lines of $\mathrm{W}$ and the polygon $\Gamma$.

From the above, it should be remarked that generalizing DIFF selectively to partial differences and SUM selectively to row sums and column sums, and using $\uparrow$ as an "up" shift and | as a "down" shift to add to LS and RS already available, we have appropriate operand and operator representations to work with discrete methods for partial differential equations in two variables; these comments hold both for Marching problems and Relaxation problems. Furthermore, transformations of data representations are easily effected. For example. let:

$$
\mathrm{Z}=\left(\zeta_{1}, \zeta_{2}, \ldots \zeta_{1}\right) \varepsilon \mathrm{II} \mathrm{REAL}
$$

and

$$
H=\left(h_{j k} \mid j=1, \ldots, n ; k=1, \ldots, m\right)
$$

then on III CPLX,

\section{LOAD III REAL H EVAL II REAL Z}

results in a complex array consisting of $I$ each arc being a contour of $Z=H(x, y)$ discretely approximated. That is, the above composition of operators constructs a contour map of $\mathrm{H}$ at elevations defined by the component of $\mathrm{Z}$.

\section{B. Problems for III CPLX}

In many respects, the generalization of II CPLX to III CPLX is simpler and more apparent than the extension of II REAL to III REAL. The reason for this rests in the fact that II CPLX already involved plane considerations, and extensions to III CPLX essentially iterates the definitions if II CPLX. Thus, for example, let $F$ be an analytic function over a domain $D$ with boundary $\Gamma$. Let $\zeta_{1}, \zeta_{2}, \ldots \zeta_{\mathrm{m}}$ be polygonal arcs in $\mathrm{D}$.
Choose the III CPLX operand

$$
\begin{array}{ll} 
& \mathrm{Z}=\left(\zeta_{1}, \zeta_{2}, \ldots \zeta_{1}\right) \\
\text { then } & \mathrm{F}(\zeta)=\mathrm{F}\left(\mathrm{F}\left(\zeta_{1}\right), \mathrm{F}\left(\zeta_{2}\right), \ldots, \mathrm{F}\left(\zeta_{1}\right)\right)
\end{array}
$$

is the result of applying the "operation" $F$ to $\mathrm{Z}$ and is an image arc representation. If $D$ is well spanned by $Z$, that is, if each point of $D$ is sufficiently near some points of $Z$, then $F(D)$ is well spanned by $F(\zeta)$ because $F$ was presumed to be analytic on the closure of $D$. If $F$ is univalent over $D$, then $F(\zeta)$ is a discreet representation of the conformal mapping defined by $F$.

As mentioned in Section 2, III CPLX EVAL achieves an appropriate meaning. For example, suppose

$$
\mathrm{V}=\left(\mathrm{v}_{1}, \mathrm{v}_{2}, \ldots, \mathrm{v}_{\mathrm{l}}\right) \varepsilon \text { III CPLX }
$$

and that each $v_{j}, j=1, \ldots, I$ lies in $D$ where $D$ and $F$ are discussed above. The $F(v)$ can be computed by an interpolation on III CPLX:

\section{(Z, $F(\zeta))$ EVAL V}

in which each point $v_{j k}$ is located in $\mathrm{Z}$ by finding its nearest neighbors, and $F\left(v_{j k}\right)$ is computed from the function values corresponding to these neighbors.

\section{Considerations for Category IV REAL and CPLX}

There are. of course, a variety of computational mathematical problems that do not fit naturally into an operator-operand format. For these, a well designed mathematical laboratory should be able to exercise library facilities and incorporate any advantageous programs, methods. etc. that exist in conventional systems into a user's own system. There is no reason to try to force an unnatural format on a given problem area. Just as this is "bad" mathematics. so is it "bad" computation. Consequently, it is important to recognize the constructive potentials and natures of mathematical endeavors and select computational approaches in resonance with these.

With this view in mind, we choose IV REAL to have operands that are symmetric matrices and represent these matrices in diagonal form. That is, our matrices are of the form:

$$
\mathrm{M}=\mathrm{U}^{*} \mathrm{DU}
$$

where $U$ is a unitary matrix whose columns are eigenvectors of $M$ and $D$ is the diagonal matrix of eigenvalues of $M$. The data structure $D$ is like a II REAL operand, and the data structure of $U$ is like a III REAL operand. Now the elementary functions on M obey:

$$
F(M)=U^{*} F(D) U
$$

and hence are computable by II REAL operations. Thus, our operational calculus extends readily to the algebra generated by $\mathrm{M}$.

For IV CPLX, Hermitian matrices follow the above in detail and require no further discussion.

For general matrices, those that are diagonalizable still 
permit reasonable structures, but the present stage of experimentation makes us prefer to include general matrices and linear systems in the mathematical library category.

From today's vantage point, it appears that mathematical laboratories have achieved a secure footing; that they provide a number of challenging computational-mathematical-design problems for interested students of modern computer sciences and mathematics.

\section{Acknowledgement}

The national government, through the Advanced Research Projects Agency, the National Science Foundation, the Office of Naval Research, and the Rome Air Development Center, have provided support to a number of the experimental mathematically oriented systems listed in the references. While this has been a small segment of the total support provided in computer research efforts, it has been a keystone of the efforts listed here. In several instances the Universities, themselves, and private industry have contributed facilities and hardware and have evidenced a lively amount of interest in these developments. At present, these systems have achieved a stage of utility that makes it important for such support to continue in order to bring a broad range of experimental usage through other campuses not necessarily involved in the early stages of these system's construction.

University of California. Santa Barbara

Santa Barbara. California

\section{References}

\section{A. Experimental Mathematical Laboratories}

1. The T.R.W. Canoga Park System

Culler, G.J., and B.D. Fried: An On-Line Computing Center for Scientific Problems, Proc. 1963 Pacific Computer Conference of the IEEE, March 1963, p. 221.

2. The S.T.L. Space Park System

Culler, G.J., and B.D. Fried: The T.R.W. Two station On-Line Scientific Computer and General Description, Computer Augmentation of Human Reasoning, Spartan Press, 1965.

3. The Columbia University System

Klerer, M, and J. May: Two-Dimensional Programming, Conference Proceedings of the Fall Joint Computer Conference 1965, Spartan Books, pp. 63-73, 1965.

4. The Amtran System

Albert, M.R., P.C. Clem, L.A. Flenker, J, Reinfelds, R.N. Seitz, and L.H. Wood: The Amtran Sampler System INstruction Manual, NASA Technical Memorandum, NASA TM X-53342, July 1966.

5. The University of California, Santa Barbara System

Culler, G.J.: The User's Manual for an On-Line System, On-Line Computing, McGraw Hill, 1967.
6. The MAP System

Kaplow, R., S. Strong J. Brachett: MAP- A System for On-Line Mathematical Analysis, MIT Publication, MAC TR 24, January 1966.

7. The Harvard System

Ruyle, A.: The Development of Systems for On-Line Mathematics at Harvard, ACM Symposium on Interactive Systems for Experimental Applied Mathematics. August 1967.

\section{B. User's Publications}

1. Culler, G.J., B.D. Fried, R.W. Huff, and J.R. Schrieffer: Solution of the Gap Equation for a Superconductor, Phys. Rev. Letters, vol. 8, p. 399, 1962.

2. Fried, B.D., and G.J. Culler: Plasma Oscillations in an External Electric Field, Phys. Fluids, vol. 6. p. 1128. 1963.

3. Schrieffer, J.R., D.J. Scalapino, and J.W. Wilkins: Effective Tunneling Density of States in Superconductors, Phys. Rev. Letters, vol. 10. p. $336,1963$.

4. Johnson, Kenneth, and Marshall Baker: Quantum Electrodynamics, Phys. Letters, vol. 11, p. $518,1963$.

5. Cheng, Hung, and David Sharp: Formulation and Numerical Solution of Sets of Dynamical Equations for Regge Pole Parameters, Phys. Rev. Letters, vol. 132, p. 1854, 1963.

6. Field, E.C., and B.D. Fried: Solution of Kinetic Equation for an Unstable Plasma in an Electric Field, Phys. Fluids, vol. 7, p. 1937, 1964.

7. Bullock, D.L., : Exchange Ratio in $\mathrm{CuF}_{2} 2 \mathrm{H}_{2} \mathrm{O}$ TRW/STL Report 9891-6001-RU-000, April 1965.

8. Dixon, W.J., : $\triangle \mathrm{V}$ to Enter Orbit About Mars, TRW IOC VM-2, April 1965.

9. DeNuzzo, J.: On-Line Solution of 2-D Trajectory Equations, TRW/STL Report 9801-6013-TU-000, May 1965.

10. Nishinago, R.G.: Preliminary Design Considerations for a Gyro-damped Gravity Gradient Satellite, TRW/STL Report 8427-6005-RU-000, May 1965.

11. Margulies, R.S.: Response of a Peak-reading Instrument to a Contaminated Signal, TRW/STL Report 9990-6963-TU-000, June 1965.

12. Pate, N.C., and S.M. Zivi: An Analysis of the Efficiency of Elliots Liquid Metal MHD Energy Conversion Cycle and IT Applicability to the Power Range of 3 to $30 \mathrm{KWE}$, TRW Systems Report 9806-602-MU-000, July 1965. 
13. Fried. B.D.. and L.O. Heflinger: Scaling Law for MHD Acceleration. TRW Systems Report 9801-6014-RU-000. July 1965.

14. Fried. R.D., and S.L. Ossakow: The Kinetic Equation for an Unstable Plasma in Parallel Electric and Magnetic Fields, UCLA Report R-3 Plasma Physics Group, November 1965.

15. Fried, B.D. and A.Y. Wong: Stability Limits for Longitudinal Waves in Ion Beam-Plasma Interactions, TRW Systems Report 9801-6015-RU-000. August 1965.

16. Coward. D.J.: Exact Inversion of Plasma Dispersion Relations. Phys. Fluids.

17. Collins. L: The Calculation of Unpaired Electron Density on the Nucleus of Many-electrons with a Thomas-Fermi-Dirac Potential.

18. Madon, Rabi: Superperturbation and Super-perturbation Theory Bounds on Electron Hydrogen Scattering. 